\title{
Creeping solitons of the complex Ginzburg-Landau equation with a low-dimensional dynamical system model
}

\author{
Wonkeun Chang, Adrian Ankiewicz*, Nail Akhmediev \\ Optical Sciences Group, Research School of Physical Sciences and Engineering, The Australian National University, Canberra, ACT 0200, Australia
}

Received 26 September 2006; accepted 1 October 2006

Available online 6 October 2006

Communicated by V.M. Agranovich

\begin{abstract}
We study creeping solitons of the complex Ginzburg-Landau equation (CGLE) using numerical simulations and analyze them with a lowdimensional model using the method of moments. We find the regions of existence of creeping solitons in the parameter space of the CGLE. We also provide a comparison with exact results obtained using numerical simulations.

Crown Copyright @ 2006 Published by Elsevier B.V. All rights reserved.
\end{abstract}

PACS: 04.30.Nk; 05.45.Yv; 42.65.Sf; 42.65.Tg

Keywords: Dissipative soliton; Method of moments; Ginzburg-Landau equation

\section{Introduction}

A creeping soliton is a special type of pulsating localized solution that changes its shape periodically and shifts a finite distance in the transverse direction after each period of oscillation. The value of the shift is constant for each period so that the soliton has a finite average velocity, although the motion occurs as a step-by-step translation in one direction. In most cases, solitons that move in this way have long flat-top profiles that consist of two fronts at the sides of the soliton. The two fronts move asymmetrically in time, thus creating creeping movements of the whole 'worm-like' formation.

Creeping solitons were first observed in numerical simulations in [1]. Their existence, for various dissipative systems, has been confirmed in later publications $[2,3]$. There is no technique that would allow us to describe pulsating solutions using exact solutions of the governing equation. Consequently, we have little hope of finding analytical solutions for creeping solitons. However, some approximations can be used to investigate the properties of creeping solitons using finite-dimensional models.

\footnotetext{
* Corresponding author.

E-mail address: ana124@rsphysse.anu.edu.au (A. Ankiewicz).
}

In particular, the method of moments has been applied to the CGLE to model pulsating localized solutions of the CGLE. This method allows us to reduce the dimensionality of the dynamical system and write the equations of motion for the parameters of the dissipative solitons. As a particular result, it allows us to estimate the period of the pulsations. In this work, we extend this method to include in the model the possibility of translational motion of localized solutions. This requires an additional variable for the velocity of the soliton, and this increases the dimensionality of the finite-dimensional dynamical system. The velocity is also a periodic function of time, and it thus describes the creeping feature of the soliton. The technique also allows us to estimate the region of existence of creeping solitons in the space of equation parameters.

\section{Cubic-quintic complex Ginzburg-Landau equation and creeping solitons}

Distributed dissipative systems demonstrate complex spatiotemporal dynamics in optical [4,5], general physical [6], chemical [7] and biological [8] systems. Spontaneous pattern formation is one of the distinctive features of nonlinear dissipative systems far from equilibrium $[9,10]$. The cubic-quintic complex Ginzburg-Landau equation (CGLE) is one of the master 


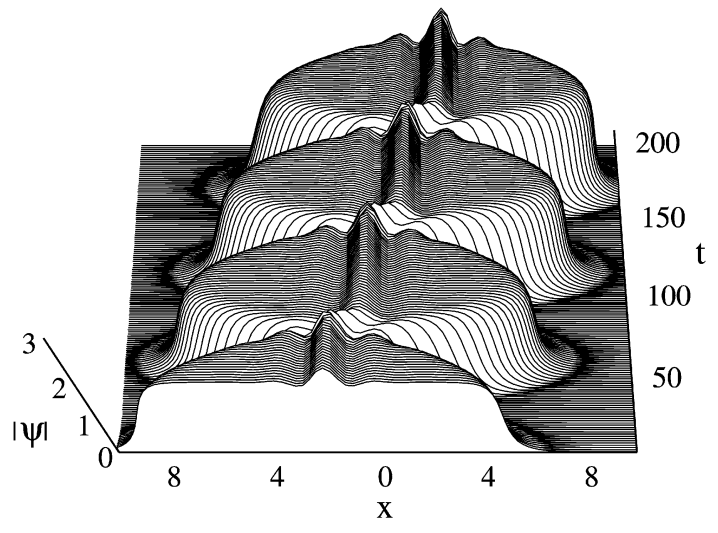

Fig. 1. An example of a creeping soliton of the CGLE. This numerical example was first found in [1]. The present simulation shows more detailed structure of creeping behavior. The asymmetric motion of the two sides of the soliton is clearly visible. The parameters of the equation are: $D=1, \delta=-0.1$, $\beta=0.101, \epsilon=1.3, \mu=-0.3, v=-0.101$.

equations for modeling such dynamics [6]. The CGLE, in dimensionless form, is written as [4]

$$
\begin{aligned}
i \psi_{t} & +\frac{D}{2} \psi_{x x}+|\psi|^{2} \psi \\
& =-v|\psi|^{4} \psi+i \delta \psi+i \epsilon|\psi|^{2} \psi+i \beta \psi_{x x}+i \mu|\psi|^{4} \psi \\
& \equiv R[\psi]
\end{aligned}
$$

where $\psi(x, t)$ is the normalized envelope of the field, $t$ and $x$ are propagation and spatial variables, respectively, $D= \pm 1$ is the dispersion coefficient, $v$ is the coefficient of the quintic nonlinearity, $\delta$ represents the linear losses, $\epsilon$ is the nonlinear gain coefficient, $\beta$ stands for the spectral filtering and $\mu$ characterizes the saturation of the nonlinear gain.

The CGLE admits localized soliton solutions which have the general name "dissipative solitons" [5]. There are various forms of dissipative solitons, including families of stationary, pulsating and exploding solitons [1,2]. A particular case of dissipative solitons is called a 'creeping soliton'. An example of a creeping soliton is shown in Fig. 1. Here the behavior is similar to that of a pulsating soliton, but, unlike a pulsating soliton, the position of the center-of-mass of the whole soliton shifts after each pulsation. Hence the soliton has an overall nonconstant velocity. A plot of the center-of-mass against $t$ shows 'jumps', so this 'reptilian' word is quite appropriate in the description of these particular solutions. Such solitons were first found numerically [1]. In this work, we study them in more detail both using numerical simulations and an approximate low-dimensional model.

\section{Region of existence of creeping solitons}

Creeping solitons exist in a certain range of the equation parameters. Previously, only isolated examples had been found [2]. Finding the complete region requires extensive numerical simulations. We have solved the CGLE using a split-step Fourier method. Thus, the second-order derivative term in $x$ of the equation is solved in Fourier space. This means that we apply periodic boundary conditions in $x$. Most of the simulations presented in the Letter were done using a numerical grid of 256 points in $x$. We used various values of step sizes along the spatial and temporal domains to verify that the results do not depend on the numerical grid size. In particular, the step size for the $t$ direction reached a minimum value 0.0002 in the most accurate runs.

Simulations have been carried out for each set of equation parameters relevant to creeping solitons. Starting from a single point in the parameter space, we can move to nearby points to confirm that creeping solitons still exist for these points. Thus, the whole region of existence can be found. An arbitrary smooth localized asymmetric initial condition was chosen as a first step in the simulations. In order for the solution to converge to the creeping soliton, the initial profile should be reasonably close to the shape of the solution. The initial parameters of the equation were chosen to be $D=1, \epsilon=1.3, v=-0.101, \delta=-0.1, \beta=$ 0.101 and $\mu=-0.3$. When the initial condition converged to a solution of the CGLE, we then varied $v$ and $\epsilon$ in order to find solutions at the new values of the parameters. Thus, step-bystep, we found solutions in a finite region of the $\epsilon, v$ plane. This technique allowed us to find the bifurcation boundaries between various types of dissipative soliton solutions.

The natural control parameter of the solution as it evolves is the total energy $Q$, which is given by the integral of $|\psi|^{2}$ over $x$ :

$Q(t)=\int_{-\infty}^{\infty}|\psi(x, t)|^{2} d x$

For a dissipative system, the energy is not conserved but evolves in accordance with the so-called balance equation [4]. If the solution remains localized, the energy evolves but remains finite. When a stationary solution is reached, the energy $Q$ converges to a constant value. If a pulse spreads out, increasing its width indefinitely, the energy tends to infinity. Another possibility is that the pulse dissipates, so that the energy then goes to zero. However, if the soliton is a pulsating one, the energy $Q$ is an oscillating function of $z$.

The results of our simulations are summarized in Fig. 2. Creeping solitons exist in the central diagonal strip of the $(\epsilon, \nu)$ plane. Below this strip, pulsations disappear and pulses are transformed into fixed shape solitons moving with constant velocity. These constant profile pulses are moving along the $x$ axis. Above the diagonal strip, the solitons are still pulsating but their average velocity is zero. Thus, the creeping feature disappears at the middle solid curve in Fig. 2. Inside the strip, the soliton energy oscillates due to the oscillations of the soliton width. These oscillations are shown in Fig. 3(a). The period of the oscillations varies in the range 50-60 across the strip. In each oscillation, the center-of-mass of the soliton moves by a finite increment. This step-wise motion of the center-of-mass is shown in Fig. 3(b). Due to the symmetry of the CGLE with respect to the inversion of the $x$-axis, a soliton can equally well move to the left as to the right, depending on the initial condition.

At the upper boundary of the central diagonal strip in Fig. 2, the soliton creeping behavior ceases. Translations after each pe- 
riod disappear and the soliton simply pulsates with zero centerof-mass velocity. These pulsating solitons exist in a narrower strip above the region for creeping solitons. The period of pulsations increases and becomes infinite at the upper boundary of this second strip. Above the second strip, any localized solution increases its width and transforms itself into a pair of fronts moving away from each other.

At the lower boundary of the central strip, the pulsations stop and the creeping behavior disappears. The soliton profile, as well as its energy, $Q$, become constant. The soliton moves with a constant velocity to the right or to the left. Each of the boundaries in Fig. 2 represents a bifurcation in the dynamics of the dissipative soliton.

Exact analytical solutions describing creeping solitons are not available. Thus, to gain understanding of creeping solitons, we need a relatively low-dimensional model. Such a reduced model can be obtained by applying the method of moments, as

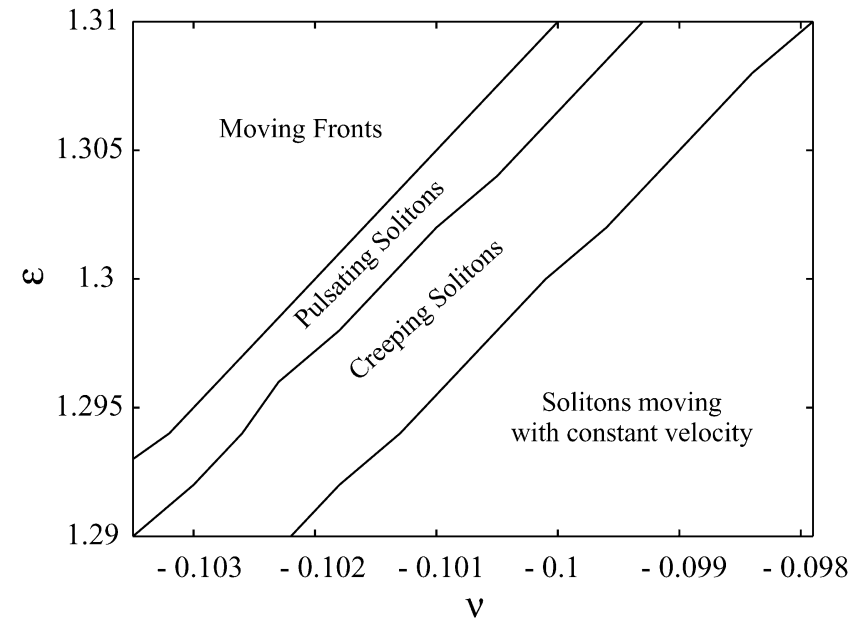

Fig. 2. Region of existence of creeping solitons found by solving the CGLE with $\delta, \beta$ and $\mu$ fixed at $-0.1,0.101$ and -0.3 , respectively. Creeping solitons exist in the central diagonal strip of this plot. Above it, there is a strip that corresponds to pulsating solitons with zero velocity. Above the upper line, solitons increase their width indefinitely, thus transforming into a pair of fronts. Below the central diagonal strip, solitons have stationary profiles and move with constant velocities. developed by Maimistov [11] to the perturbed modified nonlinear Schrödinger equation (NLSE). This method has been applied to study 'pulsating solitons' [12], where the name 'pulsating' describes the periodic contraction and expansion of the soliton's width while propagating.

\section{Reduction to finite-dimensional dynamical system}

For an arbitrary localized field $\psi$, we can introduce two integrals, namely the energy $Q$ and momentum $P$

$Q=\int_{-\infty}^{\infty}|\psi|^{2} d x$,

$P=\frac{1}{2} \int_{-\infty}^{\infty}\left(\psi \psi_{x}^{*}-\psi^{*} \psi_{x}\right) d x$,

which are two basic variables which evolve in $t$. The number of higher-order generalized moments is infinite, but we can limit ourselves to a finite number of them $[2,13]$. To study the creeping effect with the reduced model, we need five generalized moments. Three higher-order generalized moments are given by the following expressions [11]:

$$
\begin{aligned}
& I_{1}=\int_{-\infty}^{\infty} x|\psi|^{2} d x, \\
& I_{2}=\int_{-\infty}^{\infty}\left(x-x_{0}\right)^{2}|\psi|^{2} d x, \\
& I_{3}=\int_{-\infty}^{\infty}\left(x-x_{0}\right)\left(\psi^{*} \psi_{x}-\psi \psi_{x}^{*}\right) d x,
\end{aligned}
$$

where $x_{0}(t)$ is the center-of-mass. Here $I_{1} / Q$ equals $x_{0}(t)$ plus another term proportional to $f(t)$. Using the original Eq. (1), one can derive the following evolution equations for the gener-

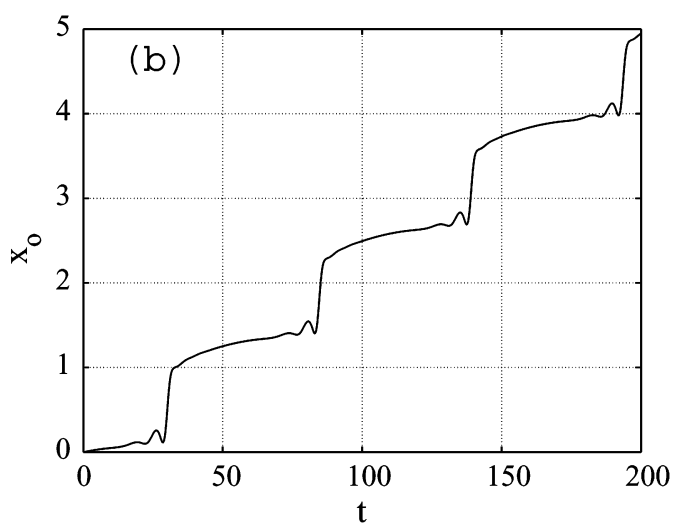

Fig. 3. (a) Soliton energy $Q$ vs. $t$ for a creeping soliton at the point $\epsilon=1.3, v=-0.101$. The energy oscillates in a wide range showing that the width of the soliton changes appreciably. (b) Center-of-mass of the same soliton vs. $t$. The soliton moves, step-by-step in the direction of increasing $x$. Due to the symmetry in $x$, the soliton could also move in the opposite direction if the initial condition was changed. 
alized moments [11]:

$$
\begin{aligned}
& \frac{d Q}{d t}=i \int_{-\infty}^{\infty}\left(\psi R^{*}-\psi^{*} R\right) d x \\
& \frac{d P}{d t}=-i \int_{-\infty}^{\infty}\left(\psi_{x} R^{*}+\psi_{x}^{*} R\right) d x \\
& \frac{d I_{1}}{d t}=i D P+i \int_{-\infty}^{\infty} x\left(\psi R^{*}-\psi^{*} R\right) d x \\
& \frac{d I_{2}}{d t}=-i D I_{3}+i \int_{-\infty}^{\infty}\left(x-x_{0}\right)^{2}\left(\psi R^{*}-\psi^{*} R\right) d x \\
& \frac{d I_{3}}{d t}=2 P \frac{d x_{0}}{d t}+i \int_{-\infty}^{\infty}\left(2 D\left|\psi_{x}\right|^{2}-|\psi|^{4}\right) d x \\
&+2 i \int_{-\infty}^{\infty}\left(x-x_{0}\right)\left(\psi_{x} R^{*}+\psi_{x}^{*} R\right) d x \\
&\left.+i R^{*}+\psi^{*} R\right) d x .
\end{aligned}
$$

We use a trial function with five parameters which depend on $t$ and one which does not. Thus we obtain five ordinary differential equations (ODEs). One can see, from Fig. 1, that it is difficult to express a creeping soliton's intricate structure and pattern of motion in a mathematical way. Our trial function is designed to approximate the creeping solitons in order to establish some basic features of their nature, rather than to predict their exact shapes.

\section{The trial function: generalized Gaussian pulse}

As our numerical simulations show, a creeping soliton changes its shape during one period from a bell-shaped pulse to a flat-top pulse. Its velocity is generally a constant with an irregular ripple superimposed on it. Therefore, we consider a trial function which is a variant of a 'super-Gaussian' function

$$
\begin{aligned}
\psi(x, t)= & a(m) \sqrt{\frac{Q(t)}{f(t)}} \exp \left(-\frac{\sqrt{m} y}{f(t)}-\frac{y^{4}}{32 f(t)^{4}}\right. \\
& \left.+i\left(b(t) y+c(t) y^{2}\right)\right)
\end{aligned}
$$

where $y=x-x_{0}(t), f(t)$ is the width, $b(t)$ and $c(t)$ are the linear and quadratic part of the chirp, respectively. The constant $m$ can be chosen arbitrarily, but it is independent of $t$, while $a$ is a constant that depends on $m$. It is chosen in such a way that the total energy, $\int_{-\infty}^{\infty}|\psi|^{2} d x$, equals $Q(t)$. For example, if $m=0.008$, we have $a=0.519548$. The trial function given in Eq. (6) is deliberately chosen to be nonsymmetric in $y$, otherwise the velocity and linear chirp will approach zero after a short transient. This would mean that we would then effectively have only three ODEs, and the creeping effect could be lost. Using the above trial function, we are still able to obtain analytic results for the integrals required.

The generalized moments in Eqs. (3) and (4), for any $m$, are given by

$$
\begin{aligned}
& P=-i Q(t)\left(b(t)+4 \sqrt{m} \frac{k_{t}}{k_{a}} c(t) f(t)\right), \\
& I_{1}=Q(t)\left(2 \sqrt{m} \frac{k_{t}}{k_{a}} f(t)+x_{0}(t)\right), \\
& I_{2}=4 \frac{k_{b}}{k_{a}} Q(t) f^{2}(t), \\
& I_{3}=\frac{16 i}{3 k_{a}} f(t) Q(t)\left(3 k_{b} c(t) f(t)-\sqrt{m} k_{c} b(t)\right),
\end{aligned}
$$

where the constants $k_{i}$ are given by the following expressions:

$$
\begin{aligned}
& k_{a}=\Gamma\left(\frac{1}{4}\right) H\left(\frac{1}{2}, \frac{3}{4} ; m\right)+8 m \Gamma\left(\frac{3}{4}\right) H\left(\frac{5}{4}, \frac{3}{2} ; m\right), \\
& k_{t}=\Gamma\left(-\frac{1}{4}\right) H\left(\frac{1}{2}, \frac{5}{4} ; m\right)+2 m \Gamma\left(-\frac{3}{4}\right) H\left(\frac{3}{2}, \frac{7}{4} ; m\right), \\
& k_{b}=\Gamma\left(\frac{3}{4}\right) H\left(\frac{1}{4}, \frac{1}{2} ; m\right)+8 m \Gamma\left(\frac{5}{4}\right) H\left(\frac{3}{4}, \frac{3}{2} ; m\right), \\
& k_{c}=3 \Gamma\left(\frac{3}{4}\right) H\left(\frac{1}{2}, \frac{5}{4} ; m\right)+8 m \Gamma\left(\frac{5}{4}\right) H\left(\frac{3}{2}, \frac{7}{4} ; m\right) .
\end{aligned}
$$

In these terms, we have used the function $H(r, s ; m)$ to represent the generalized hypergeometric function ${ }_{0} F_{2}\left(\{\} ; r, s ; m^{2}\right)$.

For $m \rightarrow 0$, we thus get the expected results for a symmetric function. Now, we are in a position to take the derivatives of Eq. (7) with respect to $t$ and equate them with the right-hand sides of Eq. (5). After rearrangement, we obtain five coupled ODEs for the $t$-dependent parameters. By solving the ODEs, we obtain equations for evolution of soliton parameters. In particular, we find that solitons can have a nonzero velocity in a certain range of the original equation parameters. All soliton parameters in this region are oscillating. This solution is a limit cycle in the five-dimensional phase space. Two projections of this limit cycle into a two-dimensional plane of parameters are shown in Fig. 4. For lower values of $\epsilon$, the parametric plot tends to a round shape, and hence the velocity of the center-of-mass and the energy $Q$ are approximately sinusoidal functions of $t$. Indeed, with $\epsilon=1.29$, the velocity appears to be almost sinusoidal. For larger values of $\epsilon$, the parametric plot becomes more elongated. This kind of irregularity is more evident as $\epsilon$ reaches the upper boundary of the creeping range. The closed curve parametric plots become larger in area, since both $x_{0}^{\prime}$ and $Q$ have a larger range of oscillations as $\epsilon$ increases.

Periodic evolution of the energy $Q$ and zig-zag type motion of the center-of-mass of the soliton $x_{0}$ along $t$ are shown in Fig. 5. Qualitatively, these curves are similar to those shown in Fig. 3. Clearly, we cannot expect a better fit because of the dramatic reduction of the number of degrees of freedom when using the trial function. However, the possibility of modeling of the creeping solitons using a simple trial function is remarkable in itself. 

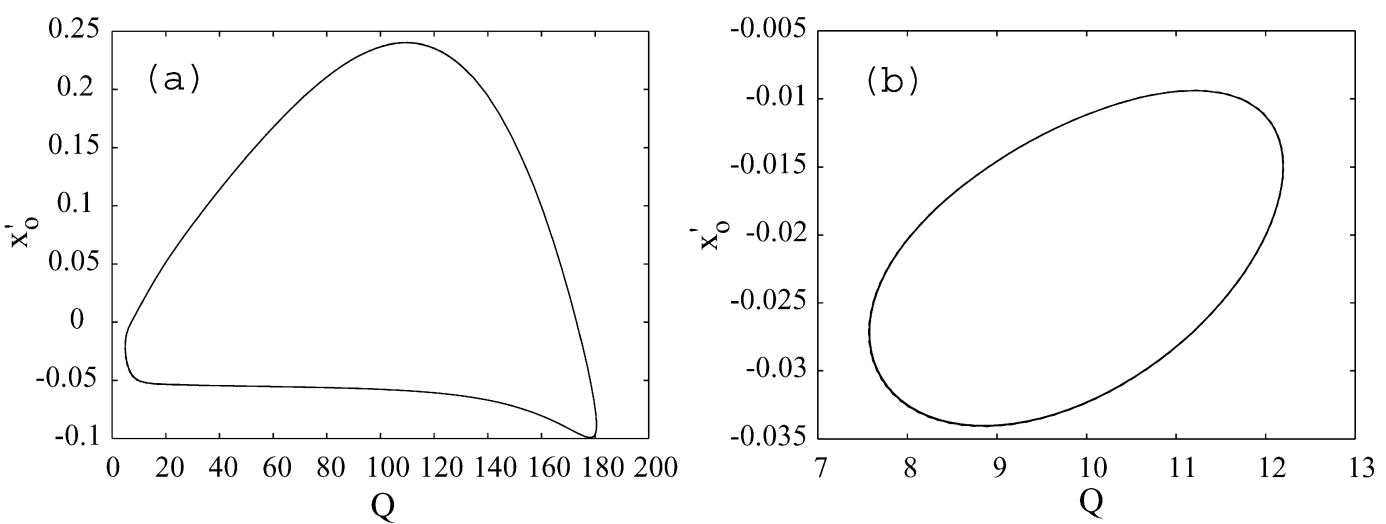

Fig. 4. Parametric plots of the soliton velocity $x_{0}^{\prime}$ versus energy $Q$ for (a) $\epsilon=1.45$ and (b) $\epsilon=1.29$. Other parameters for this calculation are chosen as $D=1$, $\delta=-0.1, \beta=0.101, \mu=-0.3, v=-0.101$, while $m=0.0025$.
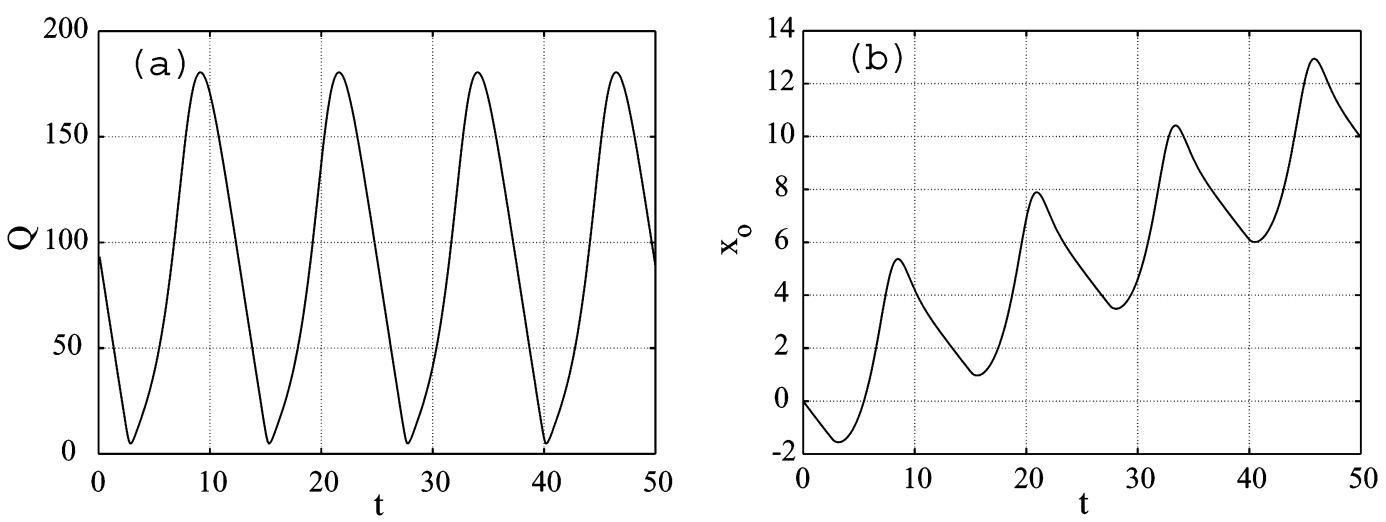

Fig. 5. (a) Soliton energy $Q$ and (b) center-of-mass $x_{0}$ along the propagation $t$ for a creeping soliton in the reduced model with $\epsilon=1.45$. Other parameters for this calculation are chosen as $D=1, \delta=-0.1, \beta=0.101, \mu=-0.3, v=-0.101$, while $m=0.0025$.

The results for the region of existence of creeping solitons in a simple approximation are shown in Fig. 6. Similar to the numerical results of Section 3, the creeping solitons occur in a central diagonal strip in Fig. 6. For lower values of $\epsilon$, the limit cycle contracts to a fixed point with all parameters including the velocity being constant. This transition is a Hopf bifurcation. Thus below the diagonal strip, we have solitons with constant shape and constant velocity in the same way as in numerical simulations.

As $\epsilon$ increases, the period of all five parameters increases. At the upper limit of existence of creeping solitons, the soliton becomes wider while retaining a constant amplitude, so the energy $Q$ becomes approximately proportional to the width. At the top of the 'creeping' range, the period becomes infinite and there is a bifurcation to solitons with constantly increasing width. This behavior corresponds to the splitting of a soliton into a pair of fronts moving away from each other in numerical simulations of Section 3. However, the velocity of the center-of-mass for this soliton is not zero, as was the case in the numerical simulations. Thus, in our reduced model we have only a single boundary separating creeping solitons from moving fronts (see Fig. 6). Again, this is hardly surprising if we take into account the drastic reduction in the number of degrees of freedom in our simplified model. Despite these discrepancies, the qualitative location of the region of existence of the creeping solitons and the boundary slopes are remarkably similar to those ob-

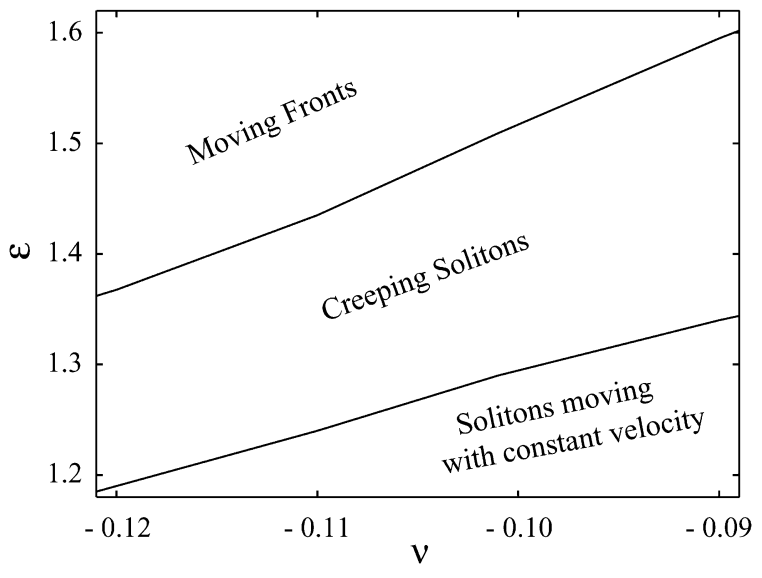

Fig. 6. Region of existence of creeping solitons in $(\epsilon-v)$ plane. Here, $D=1$, $\delta=-0.1, \beta=0.101, \mu=-0.3$, while we set $m=0.0025$.

tained in the numerical simulations. For example, in each case the lower boundary slope is $\Delta \epsilon / \Delta v \approx 4.9$.

We have studied the case $m=0.0025$ extensively, as it gives reasonably good agreement with the numerical solutions of the CGLE. The small degree of asymmetry mimics the real soliton and produces a small average velocity as the soliton creeps. For $m=0.0025, v=-0.101$, we find that the transition from fixed point to creeping soliton occurs at $\epsilon \approx 1.29$, while the bifurcation from creeping to moving fronts occurs at $\epsilon=\epsilon_{0} \approx 1.5092$. 
For fixed $v$, the parameters $Q, f, x_{0}^{\prime}, b$ and $c$ all oscillate with the same period, $T$. As we approach the upper bifurcation, we find that pulse energy, average velocity and period all increase. Numerical analysis shows that the period $T$ varies as

$$
T \approx \frac{0.6912}{\epsilon_{0}-\epsilon},
$$

so that it becomes infinite at the bifurcation point.

\section{Conclusions}

We have investigated the creeping solitons of the CGLE, both with numerical simulations of the master equation and with a reduced (5-dimensional) model. The approximate model allows us to reproduce solutions in the form of creeping solitons and to find their region of existence in the parameter space. While the reduced model gives a wider range of parameters, the qualitative behavior of the solutions is reproduced with surprisingly good accuracy.

\section{Acknowledgement}

This work is supported by the Australian Research Council.

\section{References}

[1] J.M. Soto-Crespo, N. Akhmediev, A. Ankiewicz, Phys. Rev. Lett. 85 (2000) 2937.

[2] N. Akhmediev, J.M. Soto-Crespo, G. Town, Phys. Rev. E 63 (2001) 056602.

[3] H.P. Tian, Z.H. Li, J.P. Tian, G.S. Zhou, J. Zi, Appl. Phys. B 78 (2004) 199.

[4] See, e.g., N. Akhmediev, A. Ankiewicz, Solitons: Nonlinear Pulses and Beams, Chapman \& Hall, London, 1997; G.P. Agrawal, Nonlinear Fiber Optics, Academic Press, London, 1989.

[5] N. Akhmediev, A. Ankiewicz (Eds.), Dissipative Solitons, Lecture Notes in Physics, vol. 661, Springer, Heidelberg, 2005.

[6] I.S. Aranson, L. Kramer, Rev. Mod. Phys. 74 (2002) 118.

[7] Y. Kuramoto, Chemical Oscillations, Waves and Turbulence, Springer Series in Synergetics, Springer, Berlin, 1984.

[8] M.V. Mesquita, A.R. Vasconcellos, R. Luzzi, Contemp. Phys. 40 (1999) 247.

[9] L.M. Pismen, Patterns and Interfaces in Dissipative Dynamics, Springer Series in Synergetics, Springer, Heidelberg, 2006.

[10] M.C. Cross, P.C. Hohenberg, Rev. Mod. Phys. 65 (1993) 851.

[11] A.I. Maimistov, J. Exp. Theor. Phys. 77 (1993) 727, Zh. Eksp. Teor. Fiz. 104 (1993) 3620 (in Russian).

[12] E.N. Tsoy, N. Akhmediev, Phys. Lett. A 343 (2005) 417; N. Akhmediev, J.M. Soto-Crespo, A. Ankiewicz, ACOFT 30, Sydney, 2005, p. 110.

[13] M.N. Zhuravlev, N.V. Ostrovskaya, J. Exp. Theor. Phys. 99 (2004) 427. 